\title{
A Study on the Effect of the Magnetization Direction on the Iron Loss Characteristics in Brushless DC Motors
}

\author{
Jin-Woo Jung ${ }^{1}$ and Tae Heoung Kim ${ }^{2 *}$ \\ ${ }^{1}$ Department of Electrical Engineering, Dongguk University-Seoul, Seoul 100-715, Korea \\ ${ }^{2}$ Department of Electrical Engineering, Engineering Research Institute, Gyeongsang National University, Jinju, Gyeongnam 660-701, Korea
}

(Received 26 January 2010, Received in final form 3 March 2010, Accepted 3 March 2010)

\begin{abstract}
This paper introduces two types of magnetization, and reports the effect of the magnetization direction on the iron loss in a brushless DC (BLDC) motor using a 2-D time-stepped voltage source finite-element method (FEM). The iron losses were found to consist of hysteresis and eddy current loss, which were calculated from the time variation of the magnetic field distribution. To confirm the analysis, a prototype BLDC motor was constructed with a sintered ferrite magnet. The analysis and experimental results suggest that the magnetization direction has a significant effect in terms of the iron loss characteristics of the BLDC motor.
\end{abstract}

Keywords : brushless motor, permanent magnet, finite element method, iron loss

\section{Introduction}

In recent years, brushless DC (BLDC) motors have been adopted in a range of adjustable-speed drives for energy saving, velocity or position control, and the amelioration of transients $[1,2]$. In particular, applications to home appliances, such as washing machines, refrigerators, and air conditioning systems, are increasing. BLDC motors in home appliances require high efficiency and low cost, i.e. of lower power consumption and manufacture cost. Accordingly, it is very important to predict and reduce the losses in the design process.

This paper introduces two types of magnetization and deals with the effect of its direction on the iron loss characteristics. The iron losses consist of hysteresis and eddy current losses, which were calculated from the time variation of the magnetic field distribution obtained by 2D FEM. A prototype BLDC motor was constructed to confirm the analysis. The analysis and experimental results suggest that the magnetization direction has a significant effect on the iron loss characteristic of the BLDC motors.

\section{Analysis Model and Magnetization Direction}

Fig. 1 gives a schematic diagram of the $1 / 2$ cross-sec-

*Corresponding author: Tel: +82-55-751-5349

Fax: +82-55-759-2723, e-mail: ktheoung@gnu.ac.kr tional configuration of the interior-rotor type BLDC motor. The motor has a six-pole stator and four-pole rotor.

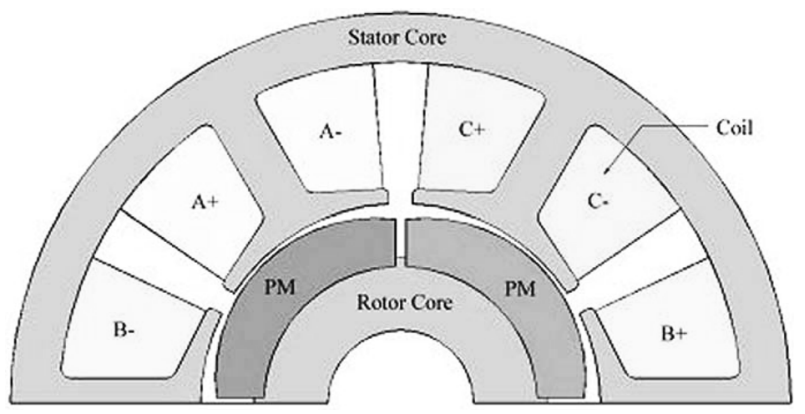

Fig. 1. Cross-section of BLDCM.

Table 1. Specifications of the BLDCM.

\begin{tabular}{clcc}
\hline \hline Section & \multicolumn{1}{c}{ Item } & Value & (Unit) \\
\hline \multirow{6}{*}{ Stator } & Number of phases & 3 & \\
& Number of slots & 6 & \\
& Outer diameter & 83 & $(\mathrm{~mm})$ \\
& Stack width & 70 & $(\mathrm{~mm})$ \\
& Number of turns/phase/pole & 77 & (turns) \\
& Parallel Circuit & 2 & \\
\hline \multirow{5}{*}{ Rotor } & Number of poles & 4 & \\
& Outer diameter & 40 & $(\mathrm{~mm})$ \\
& Magnet material & Ferrite & \\
\hline \multirow{2}{*}{ Rated } & Power & 250 & $(\mathrm{~W})$ \\
& Speed & 2900 & $(\mathrm{rpm})$ \\
\hline \multirow{2}{*}{ Air gap } & Mechanical air gap & 0.5 & $(\mathrm{~mm})$ \\
\hline
\end{tabular}



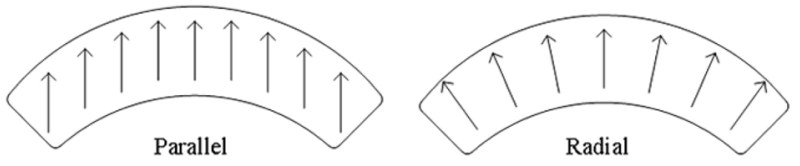

Fig. 2. Magnetization direction of the permanent magnet.

The permanent magnet material is sintered ferrite. The air gap was designed to be $0.5 \mathrm{~mm}$ to obtain a reasonable permeance coefficient value. Table 1 lists the important BLDC motor design specifications. Fig. 2 shows the magnetization direction (parallel and radial) of the permanent magnet used.

\section{Iron Loss Calculation by 2-D FEM}

The 2-dimensional governing equation for the BLDC motor is expressed as the magnetic vector potential $A$ as follows:

$$
\frac{\partial}{\partial x}\left(\frac{1}{\mu} \frac{\partial A_{z}}{\partial x}\right)+\frac{\partial}{\partial y}\left(\frac{1}{\mu} \frac{\partial A_{z}}{\partial y}\right)=-J_{0}-\frac{1}{\mu_{0}}\left(\frac{\partial M_{y}}{\partial x}-\frac{\partial M_{x}}{\partial y}\right)
$$

where

$A_{z}: \mathrm{Z}$ component of the magnetic vector potential

$J_{0}$ : Current density

$M$ : Magnetization of the permanent magnet

To consider the saturation of the magnetic material, the permeability was calculated from the B-H curve using an iterative method. The iron loss considering the harmonics can be calculated by applying the FEM to this equation.

According to Steinmetz's experimental equation, the hysteresis loss is proportional to the frequency and the $n^{\text {th }}$ power of the maximum flux density at the iron. Normally, for a commercial core, $n$ is approximately 2.0 2.5.

The eddy-current loss is also proportional to the frequency, maximum flux density, and the square of the core thickness.

In this paper, the two losses were assumed to be proportional to the square of the maximum flux density. To compensate for the difference between the real value, a proportional coefficient was included, as shown in (2), considering the difference between the calculated and real values.

$$
w_{i}=K_{e} f^{2} B_{\max }^{2}+K_{h} f \cdot B_{\max }^{2}
$$

where $f$ is the frequency, and $K_{e}$ and $K_{h}$ are proportional constants. The first and second terms are the eddy-current loss and hysteresis loss, respectively.

If (2) is divided by $f$, (3) can be obtained as a first-order equation

$$
w_{i} / f=K_{e} f B_{\max }^{2}+K_{h} \cdot B_{\max }^{2}
$$

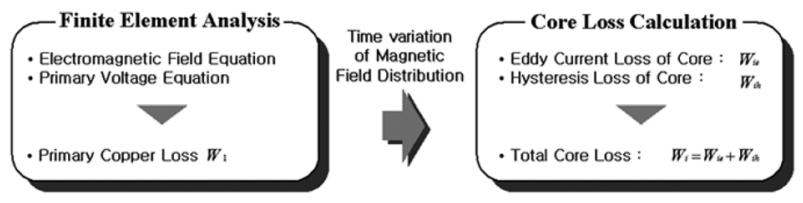

Fig. 3. Flow of loss calculation.

The approximated values of $K_{e}, K_{h}$ can be obtained by comparing this with the frequency-loss data of silicon steel.

Fig. 3 shows the calculation procedure for iron loss using the FEM.

As shown in the figure, the object was first analyzed from the variance of the distribution of the magnetic flux density using a nonlinear FEM. The copper and core losses were post processed.

\subsection{Computation of the Eddy-Current Loss Using FEM}

If the magnetic flux density $B$ of the iron varies with time, the eddy-current loss per unit mass $w_{i e}$ is shown as (4)

$$
w_{i e}=\frac{k h^{2} \sigma_{i}}{d} \frac{1}{T} \int_{0}^{T}\left|\frac{d B}{d t}\right|^{2} d t
$$

where $h$ is the thickness of the iron, $\sigma_{i}$ is the conductivity of the iron, $d$ is the density of iron, and $k$ is a proportional constant.

By using the relationship of $(1 / T) \int_{0}^{T}|d B / d t|^{2} d t=2 \pi^{2} f^{2} B_{\max }^{2}$, (4) can be rewritten as (5)

$$
w_{i e}=\frac{k_{e} d}{2 \pi^{2}} \int_{i r o n} \frac{1}{N} \sum_{k=1}^{N}\left\{\left(\frac{B_{x}^{k+1}-B_{x}^{k}}{\Delta t}\right)^{2}+\left(\frac{B_{y}^{k+1}-B_{y}^{k}}{\Delta t}\right)^{2}\right\} d v
$$

The eddy-current loss can be calculated using this equation [3].

\subsection{Computation of the Hysteresis Loss Using FEM}

It was assumed that every relative maximum and relative minimum makes a hysteresis loop and the shape of each loop is the same as the fundamental hysteresis loop. If a magnetic field with time harmonics is induced to the iron, the hysteresis loss can be calculated using the following equation $[4,5]$ :

$$
w_{i h}=\frac{k_{h} d}{T} \sum_{i=1}^{N E} \frac{\Delta V_{i}}{2}\left(\sum_{j=1}^{N_{p x}^{i}}\left(B_{m x}^{i j}\right)^{2}+\sum_{j=1}^{N_{p y}^{i}}\left(B_{m y}^{i j}\right)^{2}\right)
$$

where

$N E$ : number of finite elements of the iron;

$\Delta V_{i}$ : volume that corresponds to the $i^{\text {th }}$ finite element;

$N_{p x}^{i}, N_{p y}^{i}$ : number of relative maximum and relative 


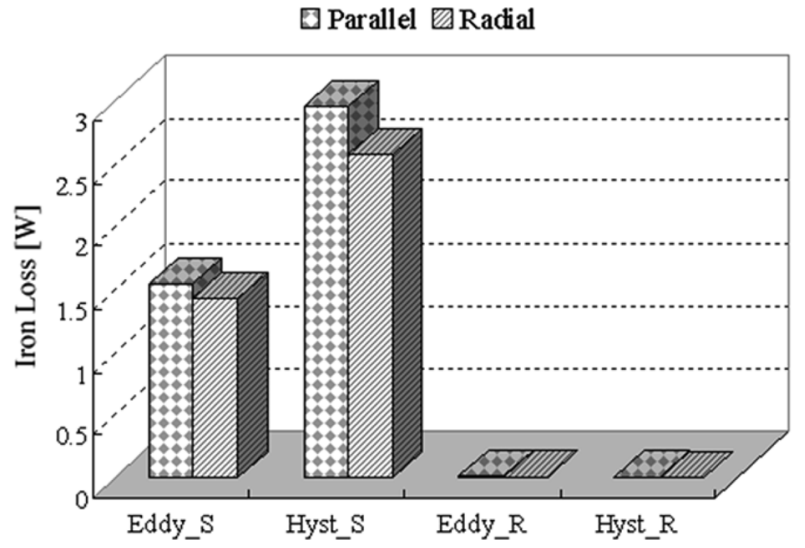

Fig. 4. Comparison of iron loss.

minimum of $i^{\text {th }}$ finite element, which has a time-varying flux in the $x, y$ directions;

$$
B_{m x}^{i j}, B_{m y}^{i j} \text { : amplitude of the hysteresis loop; }
$$

\section{Analysis Results and Discussion}

Fig. 4 shows the calculated iron loss characteristics of the BLDC motors in each magnetization direction. The iron loss in the rotor is very small in each direction. This is because there is no considerable flux variation and the flux density in the rotor is low, as shown in Fig. 5. Hence, it can be ignored. However, the stator iron loss of the parallel direction is much larger than that of the radial direction. This is illustrated in Figs. 6 and 7, which show the flux density of the central part of the teeth and stator yoke. The magnetic flux density under the parallel magnetization direction is larger and changes more rapidly. Accordingly, it is more advantageous to choose the radial magnetization direction for less iron loss. Fig. 8 compares

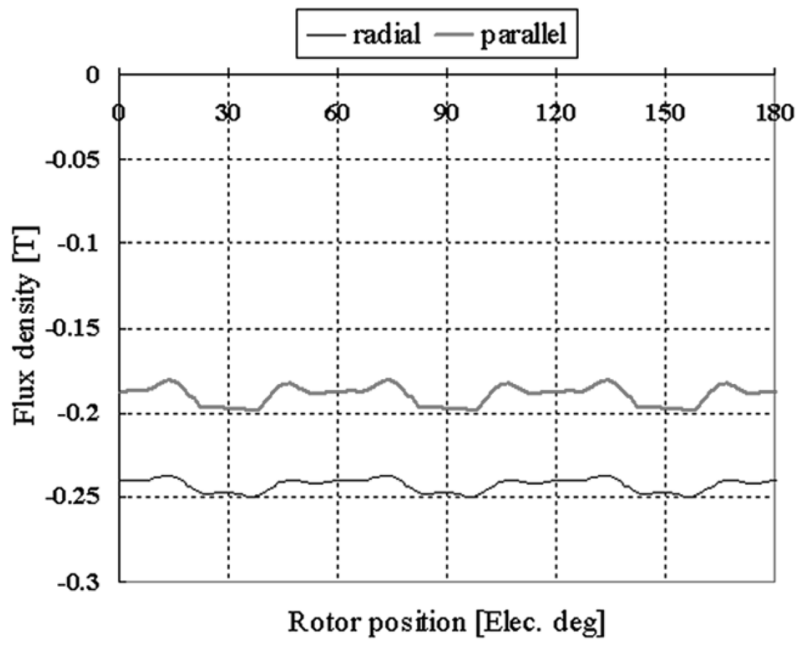

Fig. 5. Flux density variation in the rotor yoke.

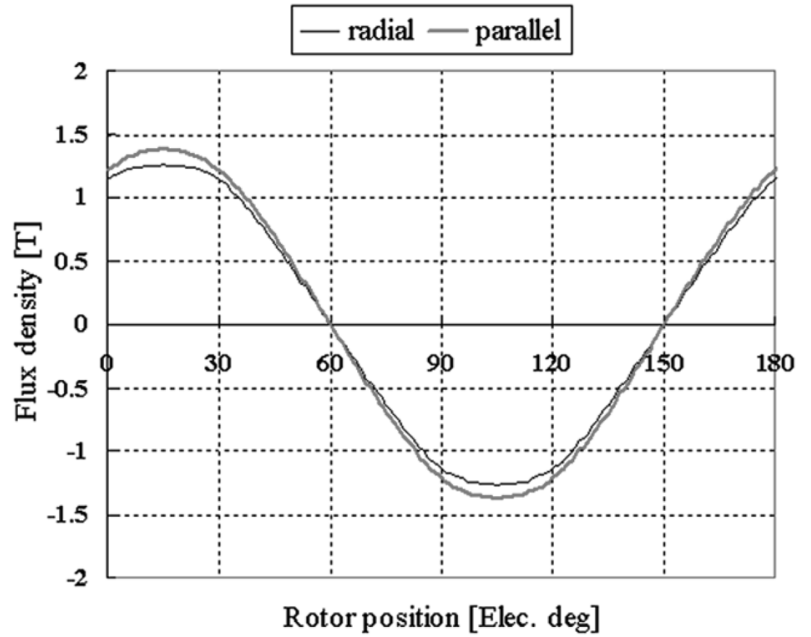

Fig. 6. Flux density variation in the stator teeth.

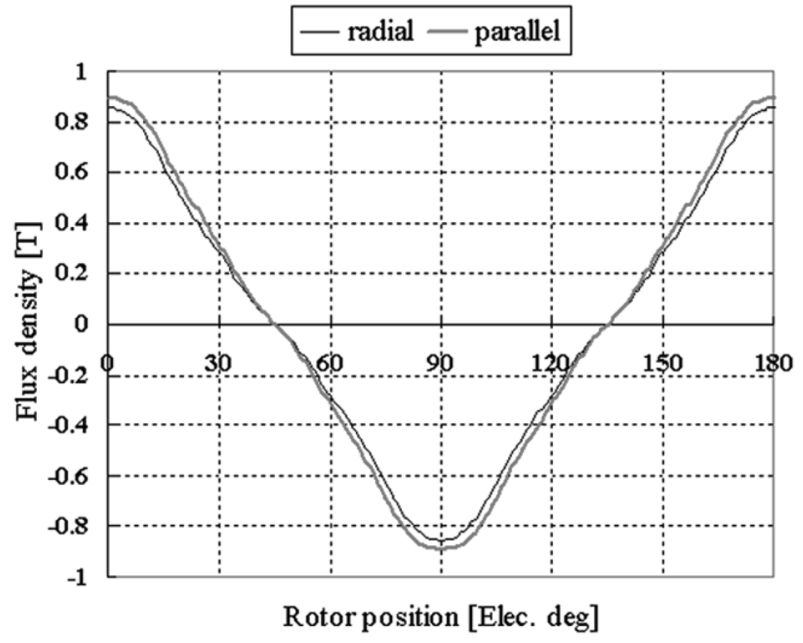

Fig. 7. Flux density variation in the stator yoke.

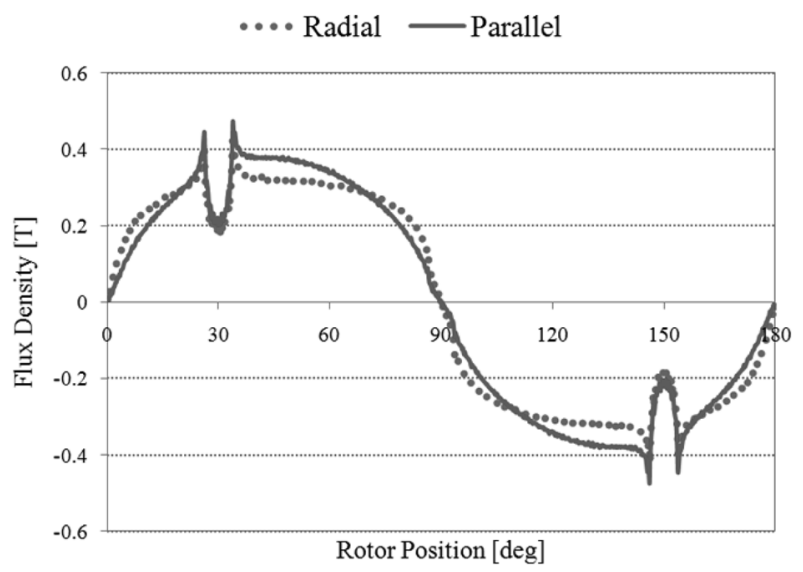

Fig. 8. Airgap flux density waveform.

the airgap flux density.

Figs. 9 and 10 show the iron loss density distribution in 


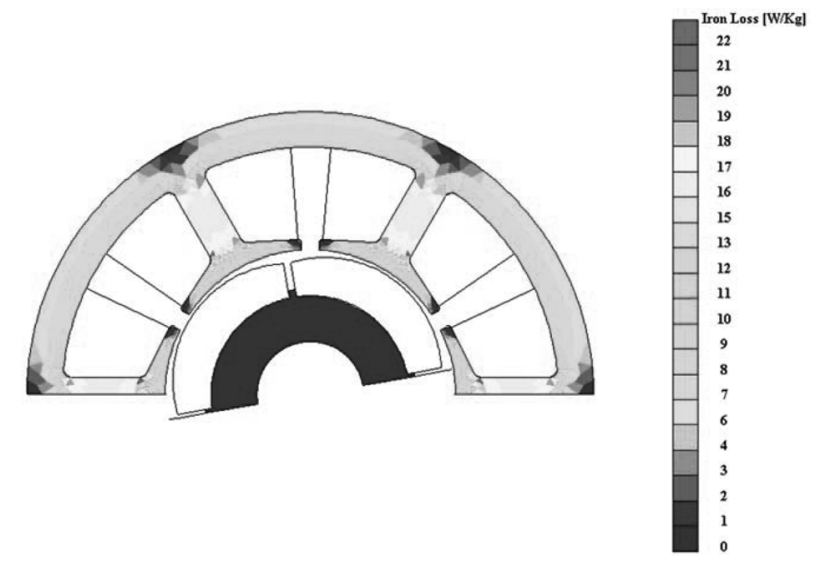

Fig. 9. Iron loss density distribution in the parallel direction.

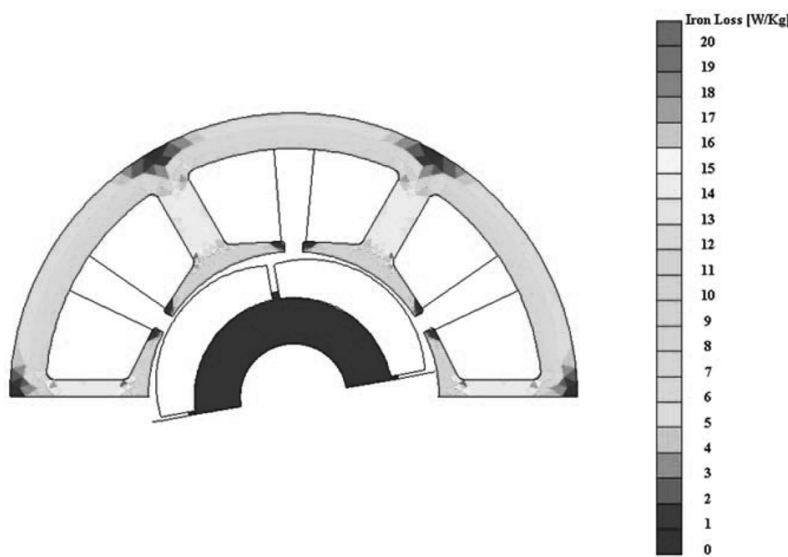

Fig. 10. Iron loss density distribution in the radial direction.

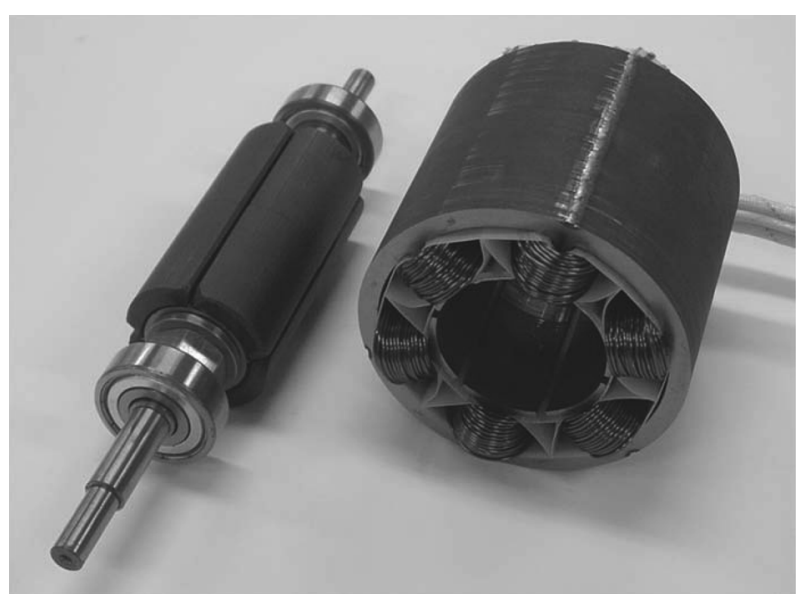

Fig. 11. Constructed prototype BLDC motor.

each direction. The iron loss in the stator is slightly higher in case of the parallel magnetization direction.

\section{Experimental Results}

To prove the analysis result, a prototype stator and rotor

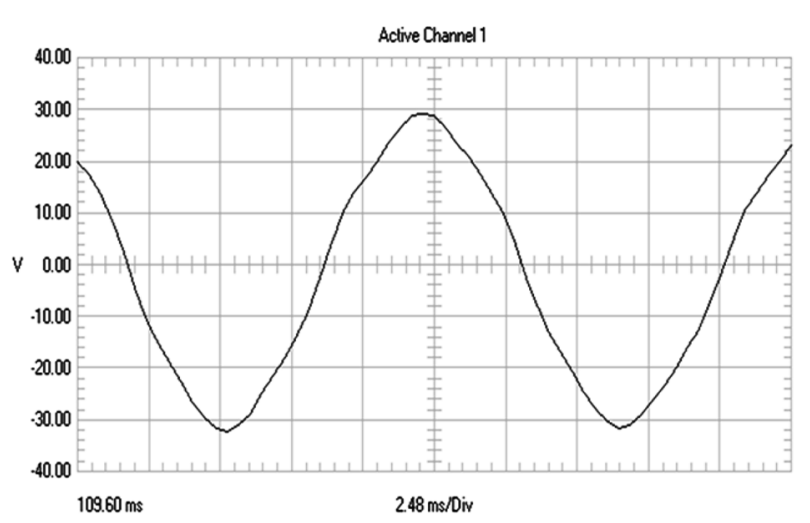

Fig. 12. Measured BEMF waveform in the parallel direction.

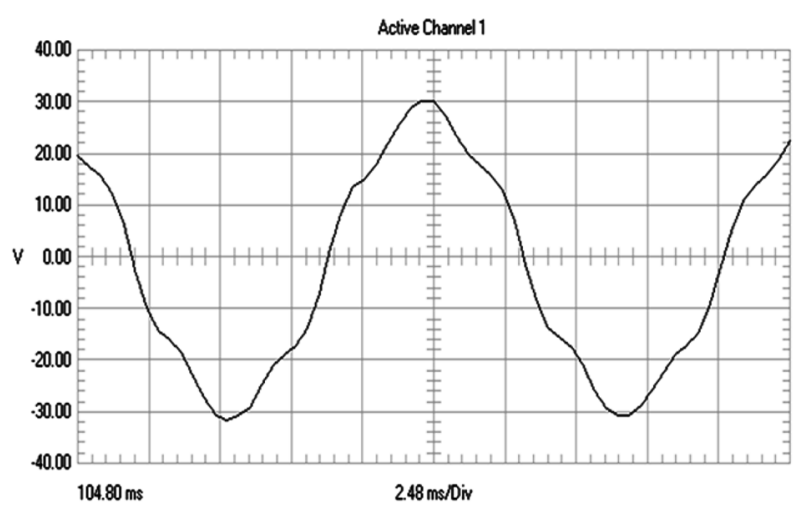

Fig. 13. Measured BEMF waveform in the radial direction.

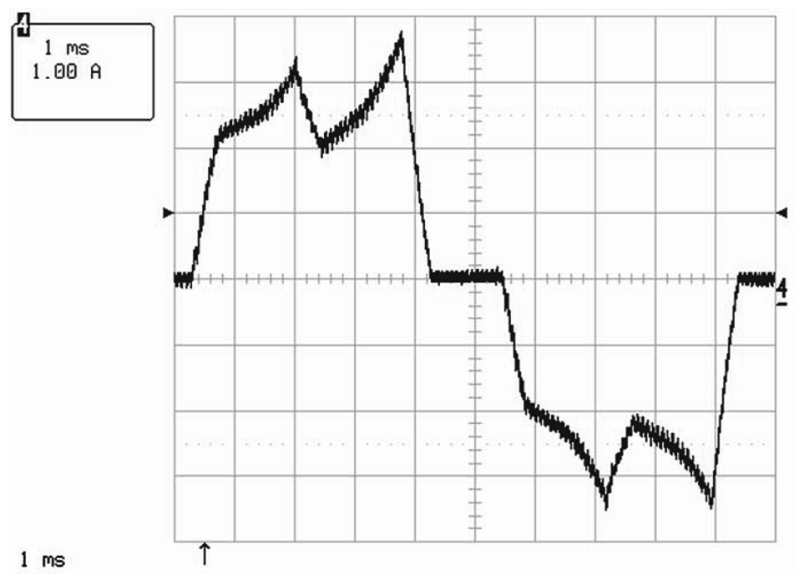

Fig. 14. Measured phase current waveform in the parallel direction.

assembly were constructed, as shown in Fig. 11. Figs. 12 and 13 show the measured line-to-line back electro-motive force $(\mathrm{BEMF})$ at $2240 \mathrm{r} / \mathrm{min}$. The measurement was carried out at no-load by driving the prototype BLDC motor using a dynamo system. The amplitude of the BEMF is similar in both cases. However, the harmonic component of the BEMF was small in parallel magnetization. This means that the torque ripple decreases in parallel magneti- 


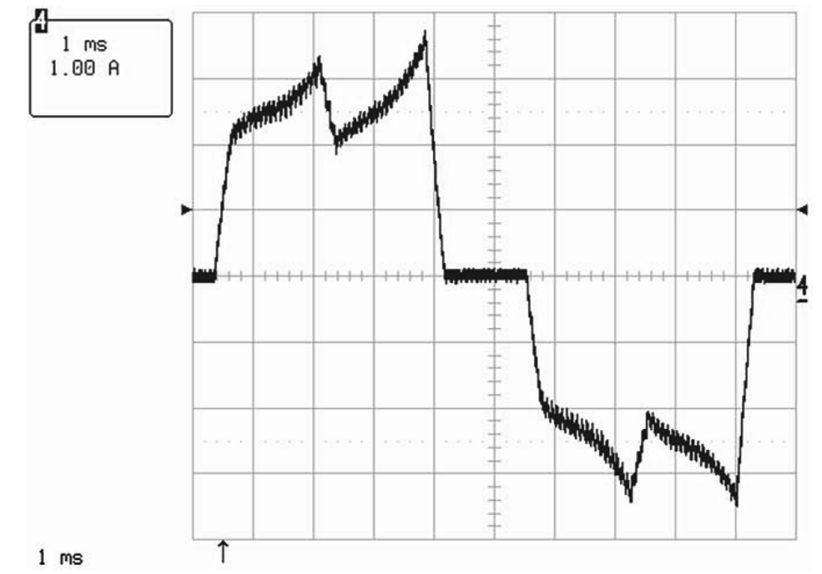

Fig. 15. Measured phase current waveform in the radial direction.

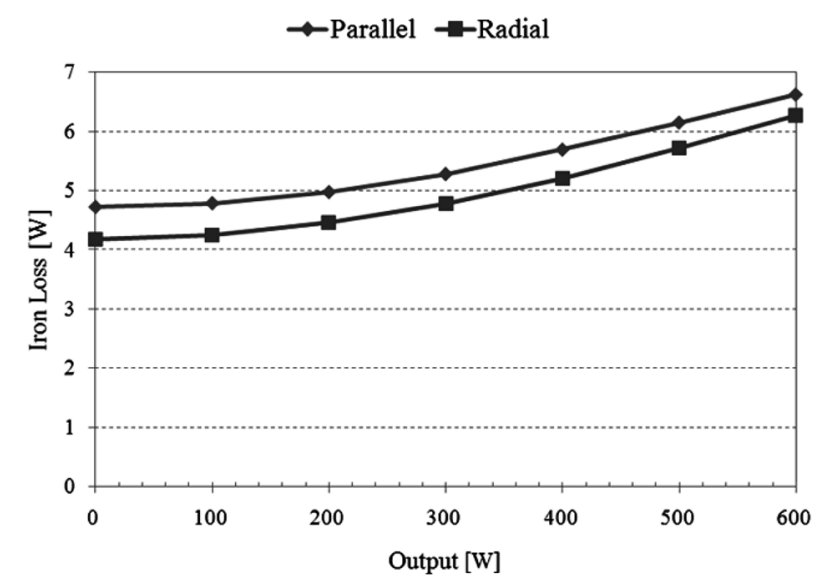

Fig. 16. Measured iron losses according to the load output. zation.

Figs. 14 and 15 compare the load current waveforms at
100 [W]. The BLDC motor was driven by alternating pulses of rectangular currents of a $120^{\circ}$ base and with a $120^{\circ}$ shift between three phases of the stator. A digital signal processor (DSP) installed controller was used to produce the pulse width modulation (PWM) patterns. The switching frequency was $12 \mathrm{kHz}$.

Fig. 16 shows the iron loss test results according to the load output. After measuring the total losses, copper, and mechanical losses, using the power and torque meter, the iron losses were obtained. The analysis and experimental results show that the radial magnetization direction is more advantageous in terms of iron loss of a BLDC motor.

\section{Conclusions}

This study compared the iron loss of the BLDC motor according to the magnetization direction of the permanent magnet. A 2-dimensional FEM was used to analyze the iron loss. The analysis and experimental results clearly show that the radial magnetization direction has merit in terms of iron loss. Accordingly, these analysis results can be used in the design of high efficiency BLDC motors.

\section{References}

[1] T. J. E. Miller, Brushless Permanent-Magnet and Reluctance Motor Drives, Oxford University Press, New York (1989) pp. 1-17.

[2] S. Kitamura, Inst. Electr. Engs. Japan 12, 1268 (1996).

[3] K. Yamazaki, IEEE Trans. Magn. 39, 1460 (2003).

[4] K. Yamazaki and Y. Haruishi, IEEE Trans. Ind. Appl. 40, 543 (2004).

[5] T. H. Kim and J. Lee, IEEE Trans. Magn. 43, 1725 (2007). 